\title{
Novel Approaches for Web-Based Access to Climate Change Adaptation Information - MEDIATION Adaptation Platform and ci:grasp-2
}

\author{
Markus Wrobel $^{1}$, Alexander Bisaro ${ }^{2}$, Dominik Reusser ${ }^{1}$, and Jürgen P. Kropp ${ }^{1}$ \\ ${ }^{1}$ Potsdam Institute for Climate Impact Research (PIK), Potsdam, Germany \\ \{wrobel, reusser, kropp\} @pik-potsdam. de \\ ${ }^{2}$ Global Climate Forum, Berlin, Germany \\ sandy.bisaro@globalclimateforum.org
}

\begin{abstract}
This paper presents two novel web-based applications for disseminating climate change adaptation related information. (i) The MEDIATION Adaptation Platform, one of the core outputs of the European FP7 research project MEDIATION (Methodology for Effective Decision-making on Impacts and AdaptaTION), offers a set of decision trees that can be browsed graphically to navigate over adaptation challenges and available types of methods for addressing them. This framework is interlinked both with a Toolbox and a set of case study descriptions. (ii) ci:grasp-2, the successor of the BMU-funded Climate Impacts: Global and Regional Adaptation Support Platform (ci:grasp), provides access to a growing pool of global and regional information on climate stimuli and impacts, as well as on adaptation projects. The paper identifies central user tasks for both applications and presents interaction metaphors that have been chosen to support users in fulfilling these tasks.
\end{abstract}

Keywords: Climate change adaptation, web-based information systems, user interfaces, HCI.

\section{Introduction}

Efficient communication of the scientific understanding of anthropogenic climate change is a prerequisite to induce the necessary shifts towards mitigation and adaptation. Against the background that such communication faces a multitude of challenges (e.g., [1-2]), web-based information systems hold some promise to facilitate the necessary dissemination of scientific information and to lower access barriers. However, designing effective information systems in the context of climate change is not a straightforward exercise due to two complimentary sets of challenges.

On the one hand, the information to be taken into account is of high complexity, ranging from climate change impacts and vulnerability to available adaptation options. In addition, existing case studies on climate change impacts, vulnerability and adaptation use different terminology and focus on different aspects of adaptation [3], which further impedes information usage. E.g., considerable work has been done to systematically characterize adaptation to climate change (e.g., [4]), including 
definition of frameworks for assessing adaptation (e.g., [5-6]), as well as towards systematic analyses (e.g., [7]) or ranking of available adaptation options (e.g., [8]). However, besides some agreement on the broad categories we are noticing the absence of a common classification scheme for adaptation options. The variety of approaches to classify adaptation originates from the inherent complexity of the field and is further complicated by the specifics of the various sectors that need to adapt to climate change. The lack of a consistently used classification scheme is not only reflected in the literature, but also in the variety of web based applications aiming to provide access to adaptation related information.

On the other hand, designing interactive climate change information supply systems faces various user interface (UI) challenges, ranging from general UI challenges (e.g., [9-10]) over challenges of web-based applications (e.g., [11-12]) to challenges related to the use of interactive maps. The latter are increasingly used to depict climate change related information. However, web-based interactive maps in general come with own usability challenges (e.g., [13]), and using maps for climate (change) communication is as well not always straightforward (e.g., [14-15]). As today there are no established standards on how to design interactive web-based applications for complex climate change and adaptation related information [16], and current webbased climate change information applications are not free of usability problems [17].

In the remainder of this paper, we present central user tasks and interaction metaphors for the MEDIATION Adaptation Platform and for ci:grasp-2, two web-based applications to be launched in 2013 that each tackle different aspects of the complex overall adaptation information supply challenge. Section 2 briefly introduces the background of both applications. Section 3 then presents central user tasks and interaction metaphors for both applications, and Section 4 concludes the paper with a brief discussion of open challenges.

\section{Central User Tasks and Interaction Metaphors for the MEDIATION Adaptation Platform}

An increasing number of methods and tools from a diverse field of disciplines is being developed to provide answers to adaptation related questions. This emerging set, however, is complex and thus not easily accessible in a systematic manner. It is the aim of the web-based MEDIATION Adaptation Platform ${ }^{1}$ for climate change adaptation methods and tools to aid users in disentangling it. To this end the MEDIATION Adaptation Platform builds on other core outputs of the MEDIATION project: a diagnostic framework for problem-oriented adaptation research, bringing together insights generated from different perspectives on adaptation into one coherent framework [18], and a Toolbox. In addition, a diverse set of case studies is made available to serve as illustrative examples of identifying and selecting salient methods and tools in adaptation research. The diagnostic framework as well as the MEDIATION Adaptation Platform provide generic methodological guidance applicable across sectors, regions and hazards for addressing vulnerability, impacts and

\footnotetext{
${ }^{1}$ http: //www.mediation-project.eu/platform/
} 
adaptation and do not aim at providing sector or regionally specific information. The intended users are scientists, policy advisors or practitioners with a technical or scientific background; the platform is not built for untrained decision-makers or the general public.

The diagnostic framework is structured along five stages: (i) assessing vulnerability (impacts \& capacity); (ii) identifying adaptation measures; (iii) appraising adaptation options; (iv) implementing adaptation actions; and (v) monitoring and evaluation. It defines a set of decision trees based on empirical and theoretical criteria to support analysts in identifying salient adaptation challenges and choosing appropriate methods to address them. The aim of the MEDIATION Adaptation Platform is to enable interactive access to this framework and efficiently interlink it with Toolbox and case study information; an intuitive and flexible navigation over the decision trees is thus one of the application's core user tasks.

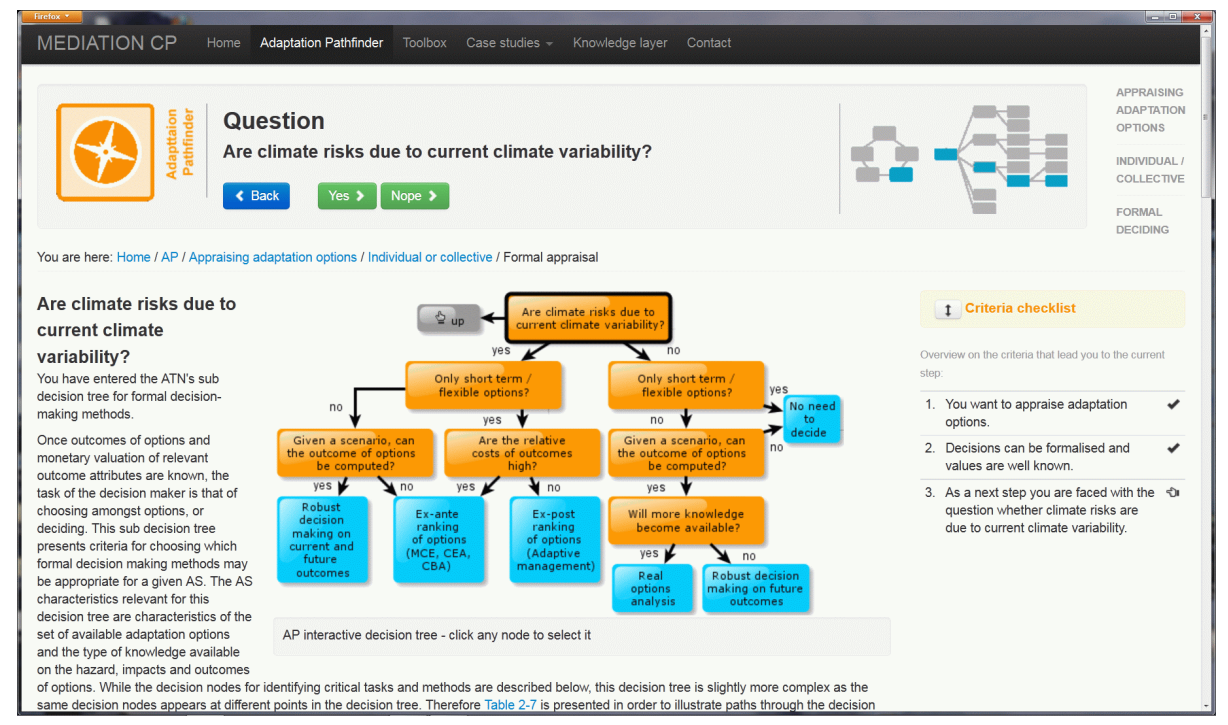

Fig. 1. Adaptation Pathfinder: Screenshot showing the sub decision tree for formal decisionmaking methods

To enable access to the diagnostic framework, the application provides the Adaptation Pathfinder. All decision trees are made available to the user in form of interactive graphic representations (Fig. 1). Due to spatial limitations and to reduce complexity, the trees are separated into and provided as set of sub-trees. By clicking a tree node, the user either (i) views available information related to the specific methodological choice or approach represented by this node, or (ii) navigates up or down in the overall decision tree structure.

To support user orientation, a combination of feedback mechanisms is applied. A popover indicating a node's content can be obtained by moving the mouse over the graphical representation of any node. A dynamically updated criteria checklist (Fig. 1, right hand) is displayed to permanently offer an overview on the choices that have led 
to the currently selected node. An additional overview diagram at the top right of the page provides further orientation aid by depicting the overall framework structure and indicating the currently selected section within this structure (Fig. 1, top right). To accommodate for different interaction preferences, the Adaptation Pathfinder offers further redundant navigation mechanisms: the trees can be alternatively browsed using answer-buttons available above the graphical representation of the trees (Fig. 1 top); in addition, shortcut lists to select a specific approach are offered. Importantly, the Adaptation Pathfinder does not prescribe any sequential access order, rather, the user can select any of the 5 stages as entry point and navigate subsequently to any sub-decision tree to focus on content of interest.

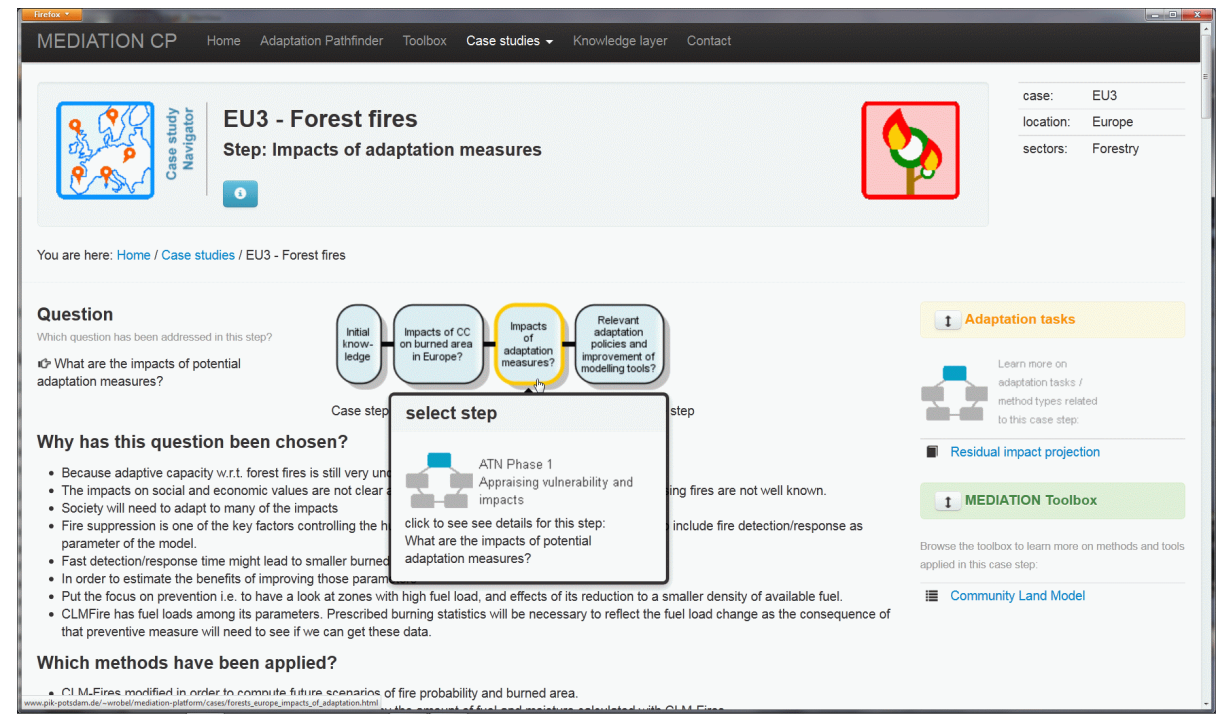

Fig. 2. Case Study Navigator showing a MEDIATION case study [19] on the effect of climate change on forest fires in Europe (screenshot)

Within the MEDIATION project, a diverse set of case studies has been conducted and informed the development of the diagnostic framework. Based on this, the MEDIATION Adaptation Platform applies a novel concept in providing access to case study information. All case studies have been broken down into a set of steps according to the diagnostic framework, with each step describing the respective question addressed, the method applied, and the results achieved.

To enable the user to flexibly browse these steps, the Case Study Navigator has been developed. Utilizing a somewhat similar interaction metaphor as applied in the Adaptation Pathfinder, interactive graphical representations are used to both depict the step structure of a case study and to allow selecting any step by clicking on its graphical representation (see Fig. 2).

A third central user task relates to integrated navigation over the three main sections of the MEDIATION Adaptation Platform (Adaptation Pathfinder, Case Study Navigator, Toolbox). To this end, while browsing through any of these three 
main sections, the user needs to be effectively supported in identifying related content within the other two. E.g., having selected a specific approach in the Adaptation Pathfinder, the user should be able to easily identify those case study steps that have applied this approach, as well as Toolbox entries related to this approach, and vice versa.

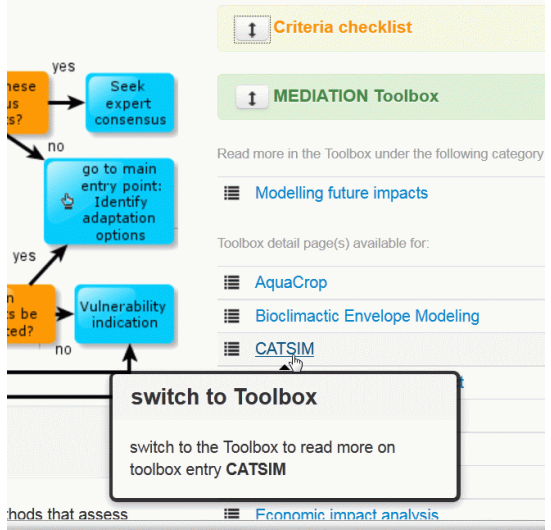

b

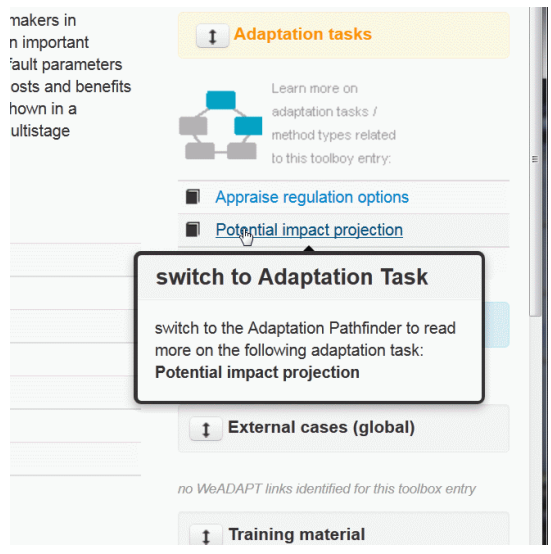

Fig. 3. Interlinking of and switching between main content sections using link boxes: (a) Adaptation Pathfinder with link box for accessing toolbox entries (b) Toolbox page with link box to related nodes in the Adaptation Pathfinder (screenshot details)

To support the user in fulfilling this task, the platform interlinks its three main sections logically in form of a triangle. To this end a tagging scheme is applied that interlinks the content elements of each main section with respective content elements of the other two. Link boxes are then derived and displayed in each main section to enable navigation to the related content elements of the respective other two main sections (Fig. 3 depicts an example for interlinking Adaptation Pathfinder and Toolbox entries). Further link boxes point to external content, enabling the user to additionally access related information as well as training material in the weADAPT ${ }^{2}$ platform.

\section{Central User Tasks and Interaction Metaphors for ci:grasp-2}

The web-based Climate Impacts: Global and Regional Adaptation Support Platform (ci:grasp) ${ }^{3}$ has been initiated by the Potsdam Institute for Climate Impact Research (PIK) and the German Agency for International Cooperation (GIZ) in a project funded by the Federal Ministry for the Environment, Nature Conservation and

\footnotetext{
2 http: / /weadapt.org/

${ }^{3}$ http: / /www.pik-potsdam.de/cigrasp-2/
} 
Nuclear Safety ${ }^{4}$. ci:grasp performs as a climate information service and aims at providing sound knowledge on current and projected climate stimuli and climate impacts, as well as on adaptation options, and to integrate both global and regional information on climate change. It aims to support decision makers in developing and emerging countries to prioritise adaptation needs, and to plan and implement appropriate adaptation measures. ci:grasp-2 is the successor of this application and provides both extended content and enhanced functionalities. Core features include content organisation based on cause-effect-relations (impact chains), support for interactive visual representations of multidimensional spatio-temporal data, and a module specifically addressing the representation of city related research results.

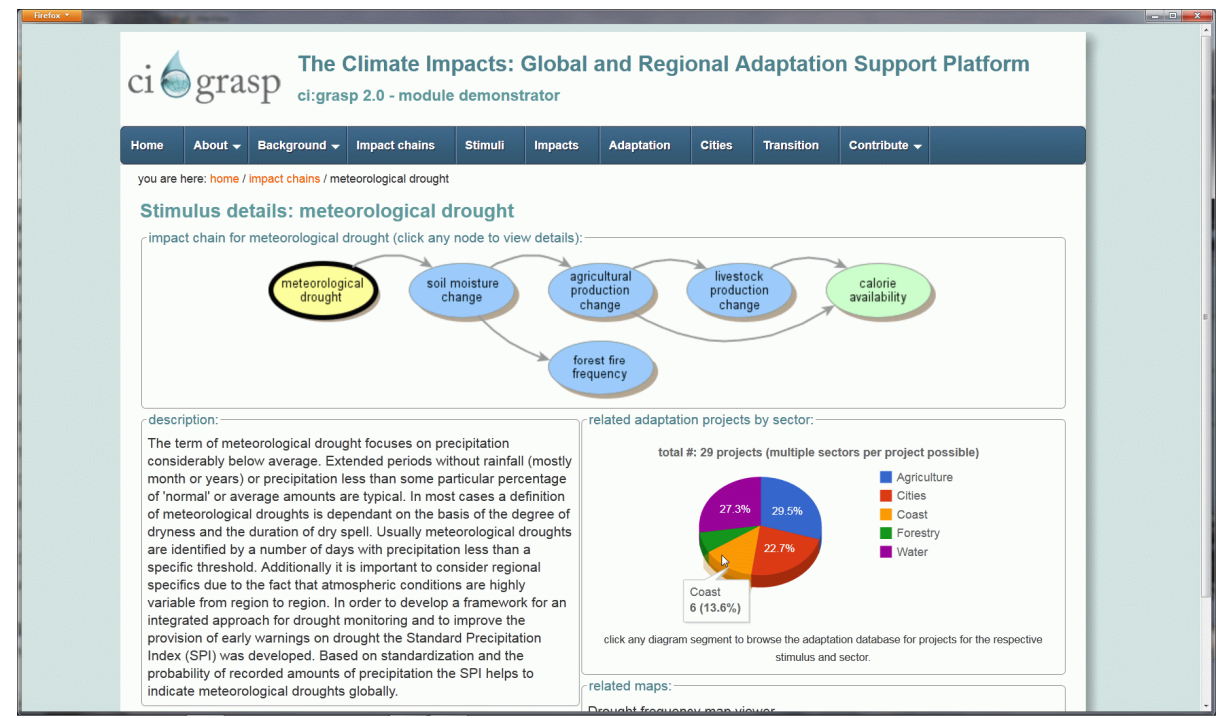

Fig. 4. Example of the interactive graphical representation of an impact chain for meteorological drought in ci:grasp-2 (screenshot)

ci:grasp-2 provides the user with information on a diverse set on climate change stimuli and impact as well as on adaptation projects. A central user task thus is efficiently gaining an overview over this complex pool of content, in order to subsequently explore it. To aid the user in the first task, the application is structured based on impact chains, i.e. simplified cause-effect-relations demonstrating how a given climate stimulus propagates through a system of interest via direct and indirect impacts it entails. cigrasp-2 offers interactive graphical representations of impact chains as means to navigate over the content in a structured manner (Fig. 4). Colour coded nodes depict a climate stimulus together with its potential impacts, as well as potentially affected livelihood conditions. By clicking the graphical representation of any node in this graphical representation the user gains immediate access to respective descriptions as well as to an overview on available content, covering respective

\footnotetext{
${ }^{4}$ Bundesministerium für Umwelt, Naturschutz und Reaktorsicherheit (BMU).
} 
stimulus or impact maps as well as related entries from a database of adaptation projects.

To provide a vivid overview on the latter, an interactive pie chart diagram displays the sectoral distribution of available adaptation projects related to a currently selected stimulus or impact (Fig. 4, bottom right). By clicking the diagram segments the user obtains a sortable result table providing a more detailed overview on the currently selected projects by title, location, approach and scale. This overview can then be used to access the detailed descriptions for every project, as well as adjusted by applying different filter criteria. Exploration of the available map content is supported by the impact chains in a similar manner. For each stimulus or impact selected, an overview over available related maps is provided; any of these entries can then be selected to be displayed in ci:grasp-2's interactive map viewer. Here, the user can subsequently zoom and pan to areas of interest as well as select different maps by applying the map viewer's filter functionality.

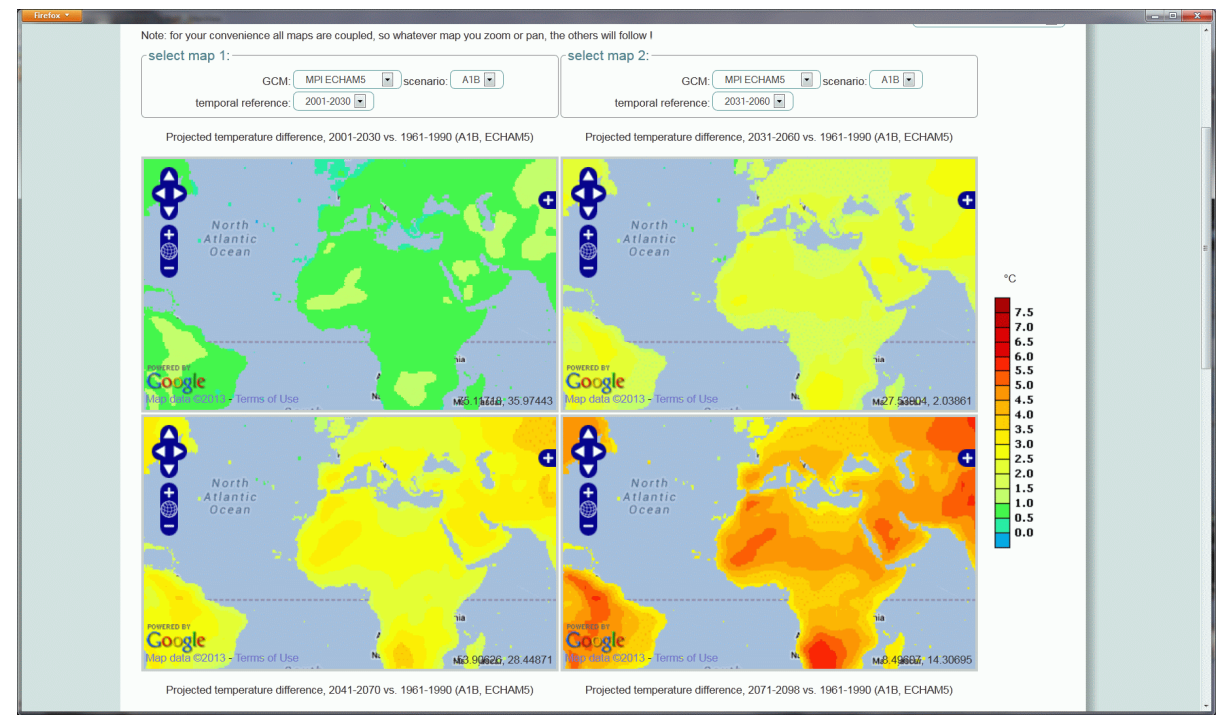

Fig. 5. Multi-map viewer showing temperature difference projections for four time slices for SRES A1B generated by ECHAM5 (screenshot detail)

De facto standards for interactive selecting of map content to be subsequently displayed on interactive maps can as today not be observed across different climate change information applications [16]. To support the user in selecting maps from a diverse pool of content, ci:grasp-2 utilizes a hierarchical multi-level set of dynamically updated UI controls. Any selection in a control of a higher level (e.g., the spatial reference) updates the selection options made available in the subsequent dependent control (e.g., the variable to be displayed), which in turn updates the next level (e.g., GCM), etc. This mechanism ensures that only meaningful attribute combinations can be selected by the user and prevents ending up with 'empty' filter results. The concept is applied both for a central map viewer providing access to ci:grasp-2's overall map 
pool, and for a set of further map viewers tailored to support accessing multidimensional information. The latter viewers combine a set of adjacent interactive maps in tabular form such that the user can jointly display characteristics of various user selectable dimensions (e.g., different GCMs, scenarios, or temporal references; Fig. 5). The maps are coupled such that any zooming or panning interaction with one of the maps directly triggers all remaining maps to adjust accordingly, so that defining an identical spatial reference for the complete set of maps is greatly facilitated. ci:grasp-2 uses this multi-map approach to represent, e.g., climate projections for AR4 and AR5 scenarios and impact projections from the ISI-MIP project ${ }^{5}$.

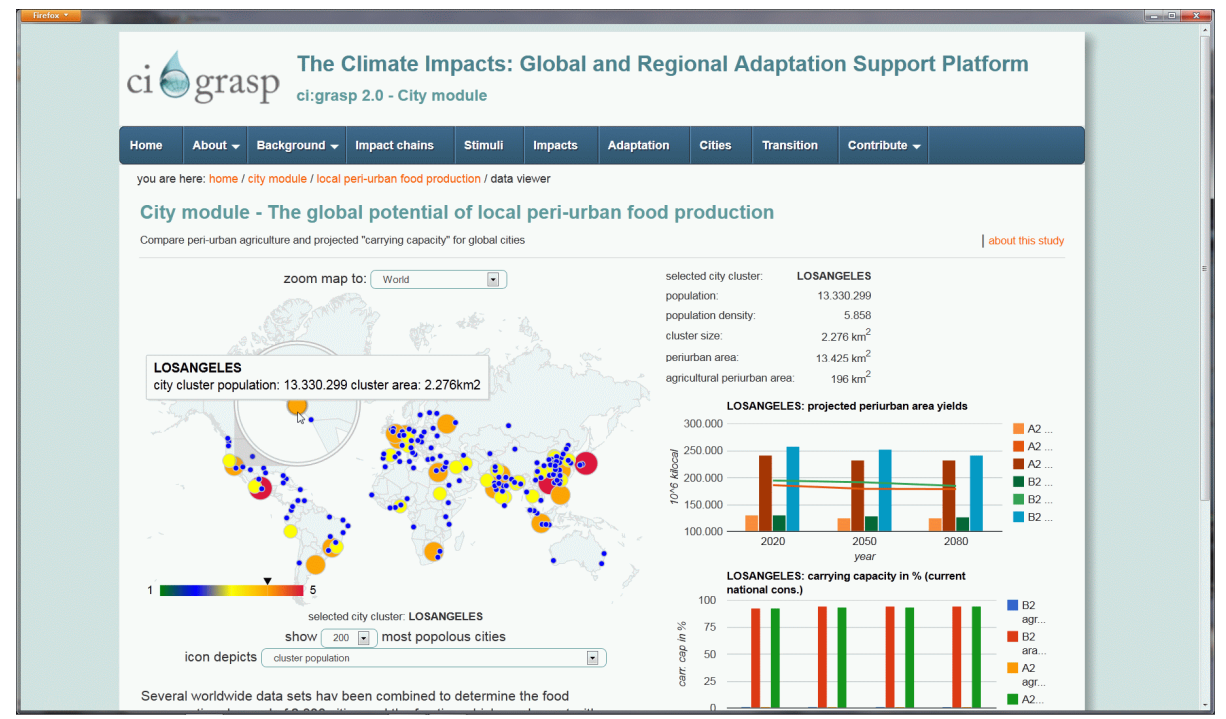

Fig. 6. Example of a tailored content viewer from ci:grasp-2's city module: Data explorer for results on the global potential of local peri-urban food production [20], allowing to compare peri-urban agriculture and projected carrying capacity for a global set of cities (screenshot)

A further core user task for ci:grasp-2 relates to flexible and intuitive exploration of a growing and necessary diverse set of research results and datasets on a variety of themes and scales. Themes to be made available comprise the transitions of dietary patterns and associated GHG emissions [21], a set of city related studies, e.g. on urban livelihood vulnerability under rapid coastal city growth [22], on city heat island effects [23], or the influence of adaptation measures during extreme heat events [24], as well as results related to adaptation to sea-level rise of the agricultural sector in Indonesia [25], a human development framework for $\mathrm{CO}_{2}$ reductions [26], Climate impacts on livelihood conditions [27] or an integrated transferable climate change vulnerability assessment [28]. To accommodate this high degree of content diversity, ci:grasp-2 refrains from a one-size-fits-all content exploration approach (which would have been desirable for the sake of simplicity, but would have come with the down-

\footnotetext{
${ }^{5}$ Inter-Sectoral Impact Model Intercomparison Project; http: / /www. isi-mip.org /
} 
side of restricted filter, representation, and comparison options for individual content subsets). Instead, the application incorporates an extensible set of tailored content viewers to display specific thematic subsets, e.g., to explore content subsections of a city module (see Fig. 6 for an example). The viewers are built using common UI APIs for interactive maps and diagrams to provide common interaction metaphors. Where possible, consistent interaction metaphors are used across different viewers to increase usability and facilitate transfer of training.

The concept of embedding an extensible set of tailored viewers allows for flexible integration of available results from climate change and adaptation research projects in an ongoing manner. Furthermore, already existing explorative tools developed within other projects can be included as well; an example for the latter is CATHY, a web-based Climate Assessment Tool for Hyderabad [29].

\section{Discussion and Conclusions}

We have identified central user tasks as well as described the respective UI metaphors of two novel climate change adaptation related web-based information supply applications. It shows that such applications need to address a range of different user tasks, ranging from browsing a set of decision trees to identify adaptation related tasks and methods (MEDIATION Adaptation Platform), to navigation over a complex set of stimulus, impact, and adaptation information and the exploration of multidimensional spatio-temporal datasets (ci:grasp-2). A universal challenge in designing appropriate interactive systems to fulfil such user tasks is the recurrent need for balancing between interface flexibility and complexity.

Extending classical UI components by appropriate interactive graphical representations can aid the user in fulfilling such tasks. However, due to as yet missing UI standards in the climate change adaptation information context, careful testing during the development should be applied to ensure that selected interaction metaphors match users' mental models. Repeated interface improvements and adjustments during development based on obtained feedback are required and desirable. E.g., feedback gathered in an earlier user test during developing the MEDIATION Adaptation Platform showed that most users judged a graphical navigation through the decision trees attractive, but some got lost in the overall structure. This led to the inclusion of an additional overview diagram to indicate overall structure and current selected section (Fig. 1, top right). After the diagram was included, it showed that some test users tried to click the nodes in this overview diagram, assuming that it could also be used for navigation. As a consequence, a next iteration included this interaction feature so that the overview diagram now provides additional 'short cuts' to the main stages and the main decision trees of the Adaptation Pathfinder.

A specific challenge in designing appropriate information applications for scientific content originates from the often required co-development of scientific content and information deploy application, due to the established approach of addressing scientific tasks within research projects with a restricted runtime. These temporal restrictions and hence the need for advancing both content and application in parallel 
can lead to initial chicken-and-egg situations between UI experts and domain experts until the required mutual understanding is achieved. A further challenge in this context relates to sustaining and updating the content of information supply applications after the respective research projects' lifetime.

Appropriate interactive web-based applications have the potential to greatly facilitate access to current knowledge on climate change and adaptation. However, further systematic research is needed to better understand which interaction approaches work best in this context, as well as the development of rigorous approaches to measure and evaluate the effectiveness of the envisaged information transfer from the scientific supply side to the demand side.

Acknowledgements. This work has been supported by the project MEDIATION (Methodology for Effective Decision-making on Impacts and AdaptaTION) (FP7, grant agreement $\mathrm{n}^{\circ} 244012$ ) and the project Methodeninventar zur Klimaanpassung (PN 10.9062.0).

\section{References}

1. Moser, S.: Communicating climate change: history, challenges, process and future directions. Wiley Interdisciplinary Reviews: Climate Change 1, 31-53 (2010)

2. Patt, A.: Communicating uncertainty to policy makers. In: Uncertainties in Environmental Modelling and Consequences for Policy Making. Springer, Heidelberg (2009)

3. Hofmann, M.E., Hinkel, J., Wrobel, M.: Classifying knowledge on climate change impacts, adaptation, and vulnerability in Europe for informing adaptation research and decision-making: a conceptual meta-analysis. Global Environmental Change 21, 1106-1116 (2011)

4. Carter, T., et al.: IPCC Technical Guidelines for Assessing Climate Change Impacts and Adaptations (1994)

5. Smit, B., Burton, I., Klein, R.J.T., Wandel, J.: An Anatomy of Adaptation to Climate Change and Variability. Climatic Change 45, 223-251 (2000)

6. Füssel, H.M.: Adaptation planning for climate change: concepts, assessment approaches, and key lessons. Sustainability Science 2, 265-275 (2007)

7. Berrang-Ford, L., Ford, J.D., Paterson, J.: Are we adapting to climate change? Global Environmental Change 21, 25-33 (2011)

8. Barr, R., Fankhauser, S., Hamilton, K.: Adaptation investments: a resource allocation framework. Mitig. Adapt. Strateg. Glob. Change 15, 843-858 (2010)

9. Shneiderman, B.: Designing the user interface: strategies for effective human-computer interaction. Addison-Wesley, Reading (1998)

10. Norman, D.: The psychology of everyday things. Basic books (1988)

11. Krug, S.: Don't make me think: A common sense approach to web usability. New Riders (2006)

12. Petrie, H., Power, C.: What do users really care about? In: Proceedings of the 2012 ACM Annual Conference on Human Factors in Computing Systems, CHI 2012, p. 2107. ACM Press, New York (2012)

13. Nivala, A.-M., Brewster, S., Sarjakoski, T.L.: Usability Evaluation of Web Mapping Sites. The Cartographic Journal 45(2), 129-138 (2008) 
14. McKendry, J.E., Machlis, G.E.: Cartographic design and the quality of climate change maps. Climatic Change 95(1-2), 219-230 (2009)

15. Ishikawa, T., Barnston, A.G., Kastens, K.A., Louchouarn, P., Ropelewski, C.F.: Climate Forecast Maps as a Communication and Decision-Support Tool: An Empirical Test with Prospective Policy Makers. Cartography and Geographic Information Science 32(1), 3-16 (2005)

16. Wrobel, M., Costa, L., Lissner, T., Moneo Laín, M., Weiß, T., Kropp, J.P.: A review of user interface conventions in web applications for climate change information. In: International Congress on Environmental Modelling and Software, Ottawa (2010)

17. Wrobel, M., Costa, L.: Web based applications for climate change related information: a usability test. In: Seppelt, R., Voinov, A., Lange, S., Bankamp, D. (eds.) International Congress on Environmental Modelling and Software. Managing Resources of a Limited Planet, Sixth Biennial Meeting, Leipzig, Germany (2012)

18. Hinkel, J., Bisaro, A.: A diagnostic framework for problem-oriented adap-tation research. Regional Environmental Change, Special Issue: Approaches for Problem-oriented Adaptation Research (in preparation)

19. Migliavacca, M., Dosio, A., Kloster, S., Ward, D.S., Camia, A., Houborg, R., Houston Durrant, T., Khabarov, N., Krasovskii, A.A., San Miguel-Ayanz, J., Cescatti, A.: Modeling burned area in Europe with the Community Land Model, J. Geophys. Res. Biogeosci. 118, 265-279 (2013), doi:10.1002/jgrg.20026

20. Kriewald, S., García Cantú Ros, A., Sterzel, T., Pradhan, P., Kropp, J.P.: Feeding cities in the post-carbon age: can peri-urban agriculture reclaim a place? (in preparation)

21. Pradhan, P., Reusser, D.E., Kropp, J.P.: Embodied greenhouse gas emissions in diets. PloS One (accepted, 2013)

22. Sterzel, T., Lüdeke, M.K.B., Walther, C., Kok, M.J.T., Lucas, P., Janssen, P., Kropp, J.P.: A global typology of urban livelihood vulnerability under rapid coastal urbanization (in preparation)

23. Zhou, B., Rybski, D., Kropp, J.P.: The statistics of urban heat island intensity (in preparation)

24. Schubert, S., Grossman-Clarke, S.: The Influence of Green Areas and Roof Al-bedos on Air Temperatures during Extreme Heat Events in Berlin, Germany, Meteorologische Zeitschrift (in press)

25. Förster, H., Sterzel, T., Pape, C.A., Moneo-Laín, M., Niemeyer, I., Boer, R., Kropp, J.P.: Sea-level rise in Indonesia: On adaptation priorities in the agricultural sector. Regional Environmental Change (2011), doi: 10.1007/s10113-011-0226-9

26. Costa, L., Rybski, D., Kropp, J.P.: A Human Development Framework for CO2 Reductions. PLoS ONE 6(12), e29262 (2011, 2012), http://www.plosone.org/article/ info\%3Adoi\%2F10.1371\%2Fjournal. pone.0029262, doi:10.1371/journal.pone.0029262

27. Lissner, T.K., Reusser, D.E., Lakes, T., Kropp, J.P.: Measuring livelihood conditions in the context of climate change and sustainability (in preparation)

28. Holsten, A., Kropp, J.P.: An integrated and transferable climate change vulnerability assessment for regional application. Natural Hazards 64, 1977-1999 (2012), doi:10.1007/s11069-012-0147-z

29. Kit, O., Lüdeke, M.K.B., Reckien, D.: Defining the bull's eye: satellite imagery-assisted slum population assessment in Hyderabad/India. In: Urban Geography 2013 (2013), http: / /www.tandfonline.com/doi/full/10.1080/02723638.2013.77 8665 